\title{
Ferramentas de gestão para enfermeiros auditores
}

\author{
Management tools for nurses auditors \\ Herramientas de gestión para enfermeros auditores
}

Recebido: 27/01/2022 | Revisado: 01/02/2022 |Aceito: 11/02/2022 | Publicado: 16/02/2022

Tatiana Souza da Silva Werle

ORCID: https://orcid.org/0000-0002-6587-6092 Universidade Federal do Estado do Rio de Janeiro, Brasil E-mail: tatiana.werle@edu.unirio.br

Antonio Rodrigues de Andrade

ORCID: https://orcid.org/0000-0002-2123-4215

Universidade Federal do Estado do Rio de Janeiro, Brasil E-mail: prof.arandrade@gmail.com

\begin{abstract}
Resumo
Objetivos: descrever e analisar por meio de referências bibliográficas a importância das ferramentas para a auditoria em saúde. Método: trata-se de um estudo de revisão integrativa. A operacionalização foi desenvolvida a partir das seguintes etapas: identificação da questão da busca, seleção dos descritores, seleção da base de dados, aplicação de critérios de inclusão e exclusão, identificação dos assuntos dos estudos selecionados e análise e interpretação de resultados. Resultados: foram identificados 10 artigos que atendiam ao critério de inclusão por estarem alinhados à questão norteadora. O conteúdo dos artigos foi analisado mediante a técnica de conteúdo de Bardin e emergiram as categorias a seguir: anotações de enfermagem, educação em saúde, gestão da qualidade, protocolos para tecnologias em saúde e protocolos clínicos e indicadores de qualidade. Considerações finais: fica evidenciada a importância de ferramentas de gestão para que enfermeiros auditores possam executar o processor de auditoria nas organizações, considerando o ambiente globalizado e cada vez mais competitivo, pois tais instrumentos possibilitam atingir metas, agilizar a análise de resultados e gerar relatórios.
\end{abstract}

Palavras-chave: Auditoria de enfermagem; Gestão em saúde; Qualidade da assistência à Saúde.

\begin{abstract}
Objectives: to describe and analyze, through bibliographical references, the importance of tools for health auditing. Method: this is an integrative review study. The operationalization was developed from the following stages: identification of the guide question, selection descriptors, database selection, application of inclusion and exclusion criteria, subjects identification of the selected studies and analysis and interpretation of results. Results: 10 articles were identified that met the inclusion criteria for being aligned with the guiding question. The content of the articles was analyzed using Bardin's content technique and emerged the following categories: nursing notes, health education, quality management, protocols for health technologies and clinical protocols and quality indicators. Final considerations: the importance of management tools is highlighted that nurse auditors can execute the audit processor in organizations, considering the globalized and increasingly competitive environment, as such instruments make it possible to achieve goals, streamline the analysis of results and generate reports.
\end{abstract}

Keywords: Nursing audit; Health Management; Quality of health care.

\section{Resumen}

Objetivos: describir y analizar, a través de referencias bibliográficas, la importancia de las herramientas para la auditoría sanitaria. Método: se trata de un estudio de revisión integradora. La operacionalización se desarrolló a partir de los siguientes pasos: identificación de la pregunta de búsqueda, selección de descriptores, selección de la base de datos, aplicación de criterios de inclusión y exclusión, identificación de los sujetos de los estudios seleccionados y análisis e interpretación de resultados. Resultados: Se identificaron 10 artículos que cumplieron con los criterios de inclusión para estar alineados con la pregunta orientadora. El contenido de los artículos se analizó mediante la técnica de contenido de Bardin y surgieron las siguientes categorías: notas de enfermería, educación en salud, gestión de la calidad, protocolos de tecnologías sanitarias y protocolos clínicos e indicadores de calidad. Consideraciones finales: se resalta la importancia de las herramientas de gestión para que las enfermeras auditoras puedan ejecutar el procesador de auditoría en las organizaciones, considerando el entorno globalizado y cada vez más competitivo, ya que tales instrumentos permiten alcanzar metas, agilizar el análisis de resultados y generar informes.

Palabras clave: Auditoría de enfermería; Manejo de la salud; Calidad de la asistencia sanitaria. 


\section{Introdução}

$\mathrm{O}$ trabalho da equipe de enfermagem tem impacto direto no serviço de auditoria, contribuindo para a medição de itens auditados, glosas e retorno financeiro. $\mathrm{O}$ instrumento principal utilizado pelo serviço de auditoria é o que descreve os cuidados, atividades, intercorrências e os procedimentos produzidos pela equipe multidisciplinar, ou seja, o prontuário do paciente (Carvalhoet al., 2010).

Como uma ferramenta de gestão, permite avaliar a eficiência, eficácia, efetividade e economicidade das ações e serviços de saúde no contexto do sistema de saúde, além de prestar cooperação técnica, propor medidas corretivas, servir de suporte ao controle social, subsidiar o planejamento e o monitoramento com informações validadas e confiáveis, sendo uma marcante contribuição para a saúde pública brasileira (Peron, 2021).

Outrossim, permite controlar a qualidade de um produto ou serviço prestado por meio da atividade de seleção de pontos críticos do processo, é possível à gerência avaliar, confirmar ou verificar as atividades relacionadas com a qualidade, constituindo um processo positivo e construtivo (Luongo, 2011).

Iniciativas no sentido de garantir a assistência de qualidade estão cada vez mais emergentes no cenário atual, seja por movimento governamental, ou por entidades independentes, por pressão social ou pelos clientes corporativos que financiam o seguro saúde, que almejam retornos concretos em face ao investimento no serviço (Scarparo et al., 2010).

Diante desta constatação, pontuou-se a necessidade de entender a concepção, os métodos, a finalidade e os parâmetros que sustentem, na prática, alinhado a interpretação das regras contratuais, de negócio e das tabelas de precificação e seus impactos sobre a auditoria de enfermagem.

Este estudo visa analisar e identificar a importância das ferramentas de gestão para a auditoria de enfermagem. Diante dessa problemática surgiu a seguinte pergunta norteadora do estudo: Como as ferramentas de apoio a auditoria contribuem para o processo de auditagem por enfermeiros.

\section{Metodologia}

Visando atender os objetivos traçados, este trabalho realizou um estudo de revisão integrativa, com intuito de relacionar o conhecimento com as pesquisas disponíveis na literatura, a respeito da temática tratada. Para tal, procedeu-se com as seguintes etapas: identificação da questão da busca; seleção dos descritores; seleção das bases de dados; aplicação de critérios de inclusão e exclusão; identificação dos estudos selecionados e análise e interpretação dos resultados (Botelho et al., 2011).

Os descritores estabelecidos para serem utilizados, após uma consulta ao "Descritores em Ciências da Saúde" - (DeCS) e (MeSH) foram: "Auditoria de enfermagem"; "Nursing Audit", "Gestão em Saúde”, "Health Management ", "Qualidade da assistência à saúde" e "Quality of Health Care". A estratégia de busca foi adaptada às bases de dados pesquisadas, seguindo seus critérios de pesquisa. Utilizaram-se os operadores booleanos "AND" e "OR". A seleção ocorreu através do metabuscador da Biblioteca Virtual em Saúde. O quadro 1 demonstra a estratégia de busca utilizada. 
Quadro 1: Estratégia de busca utilizando o metabuscador da BVS.

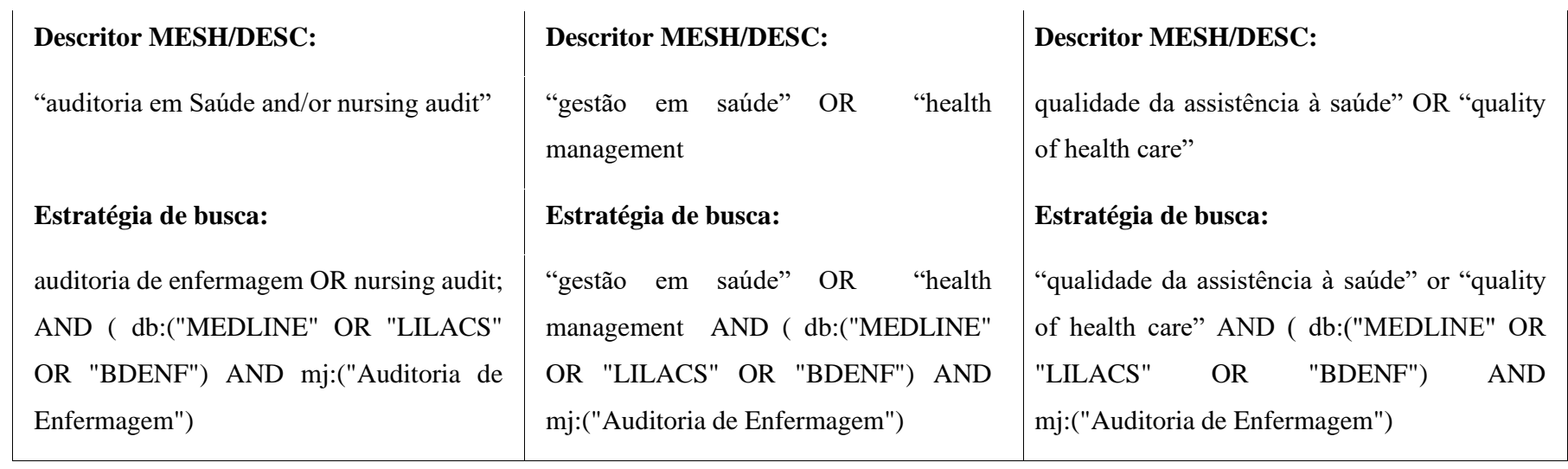

Fonte: Autores.

Os critérios de exclusão foram os estudos duplicados; os registros que não abordam o tema proposto. Os artigos científicos foram extraídos das fontes eletrônicas MEDLINE (Medical Literature Analysis and Retrieval System Online); BDENF e LILACS (Literatura Latino-Americana e do Caribe em Ciências da Saúde.O fluxograma de captação das revisões seguiu as recomendações do Preferred Reposting Items for Systematic and Meta-Analyses extension for Scoping Reviews - PRISMA-ScR (PRISMA extension for Scoping Review) (Tricco et al., 2018), conforme a figura 1 apresenta:

Figura 1 - Fluxograma da revisão integrativa, e análise das buscas nas bases de dados, 2022.

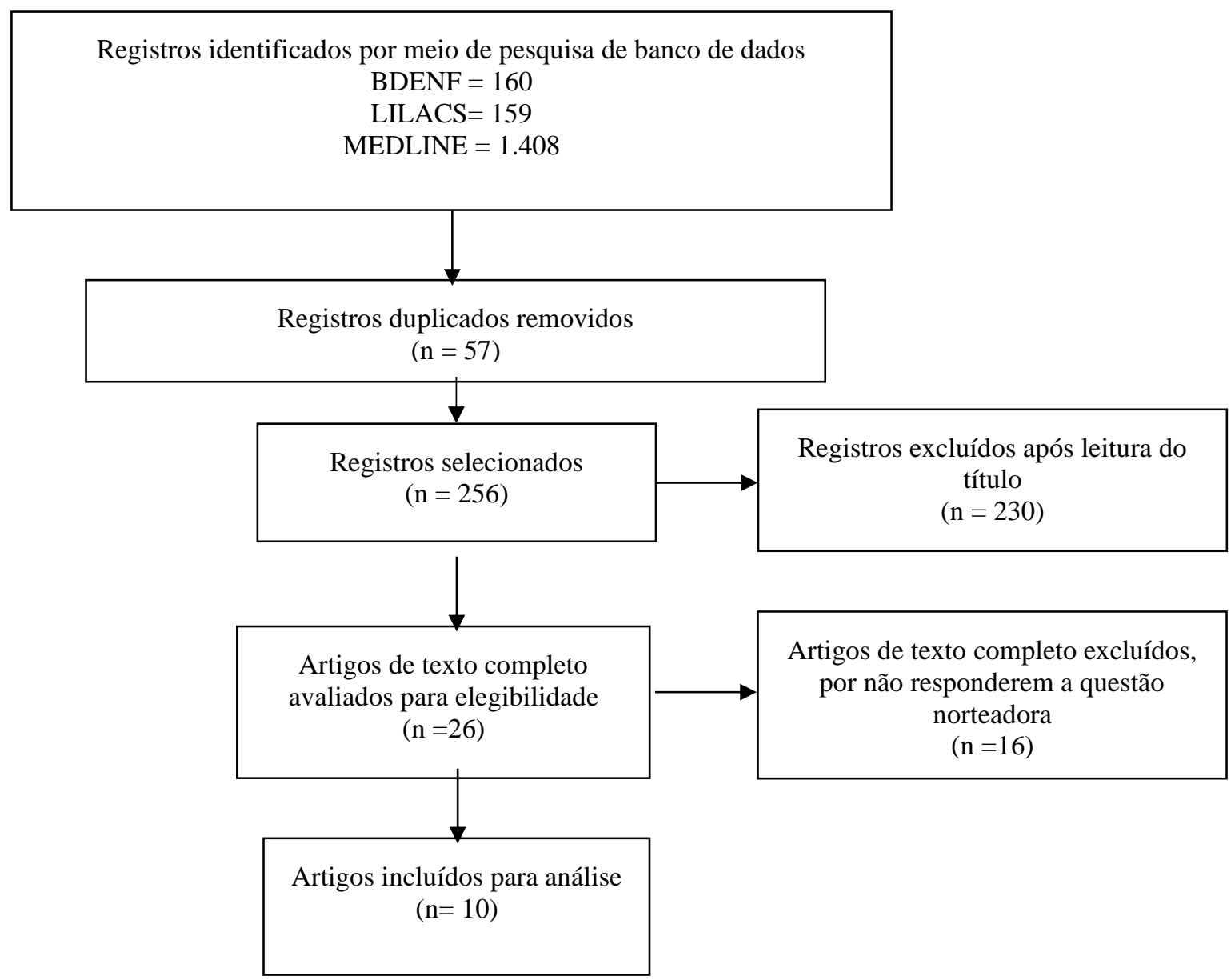

Fonte: Autores. 
O nível de evidência científica dos artigos incluídos será identificado de acordo com os seguintes critérios: Nível 1(evidências resultantes da meta-análise de múltiplos estudos clínicos controlados e randomizados); Nível 2 (evidências obtidas em estudos individuais com delineamento experimental); Nível 3 (evidências de estudos quase-experimentais); Nível 4 (evidências de estudos descritivos (não-experimentais) ou com abordagem qualitativa); Nível 5 (evidências provenientes de relatos de caso ou de experiência; e Nível 6, evidências baseadas em opiniões de especialistas) (Galvão, 2006).

Após os resultados das buscas nas bases de dados nacionais e internacionais, foram executadas as etapas do fluxograma de seleção composto por quatro passos. No primeiro passo, dois revisores (pesquisador e orientador) revisaram os títulos e resumos dos artigos para a verificação de relação à temática. No segundo passo, os artigos duplicados foram revisados para exclusão. No terceiro passo, os textos foram lidos integralmente em tela eletrônica, sendo incluídos aqueles que se referem aos aplicativos móveis de reabilitação neurológicas voltadas aos pacientes com limitação da mobilidade.

A avaliação da qualidade dos estudos na revisão foi realizada utilizando ferramentas do Programa de Competências de Avaliação Crítica (Critical Appraisal Skills Programme, CASP). O Checklist consiste em 10 questões (duas para a seleção dos estudos e oito para o plano de pesquisa, coleta e análise de dados, ética, reflexividade e implicações da pesquisa) (Oxford Brazil, 2018). Padronizou-se as três primeiras questões como sendo fundamentais e se, para qualquer uma delas, a resposta for "não", o artigo deve ser excluído.

Os artigos foram analisados mediante a técnica de análise de conteúdo de Bardin, (2011), com base na concepção de necessidade apresentada.

\section{Resultados}

Conforme critérios de inclusão, foram identificados 1.727 artigos. Após a leitura dos títulos, foram selecionados 256 artigos por estarem relacionados ao objetivo da pesquisa. Após a leitura dos resumos, excluíram-se os artigos que não respondiam à pergunta de pesquisa, totalizando 26 artigos selecionados para leitura completa. Após a leitura dos artigos selecionados, 16 foram excluídos, pois estavam relacionados à área de gestão resultando numa amostra de dez artigos, que foram discutidos e analisados.

O mapeamento dos estudos foi feito inserindo os dados em um banco de dados no Microsoft Word e classificado de acordo com as seguintes variáveis: Ano de Publicação, Nome da revista, Base de Dados, Título, Objeto, Metodologia e Evidência conforme disposto na Tabela 1:

Tabela 1 - Distribuição dos artigos segundo ano, revista, base, título, objetivo, metodologia e evidência. Rio de Janeiro. 2022.

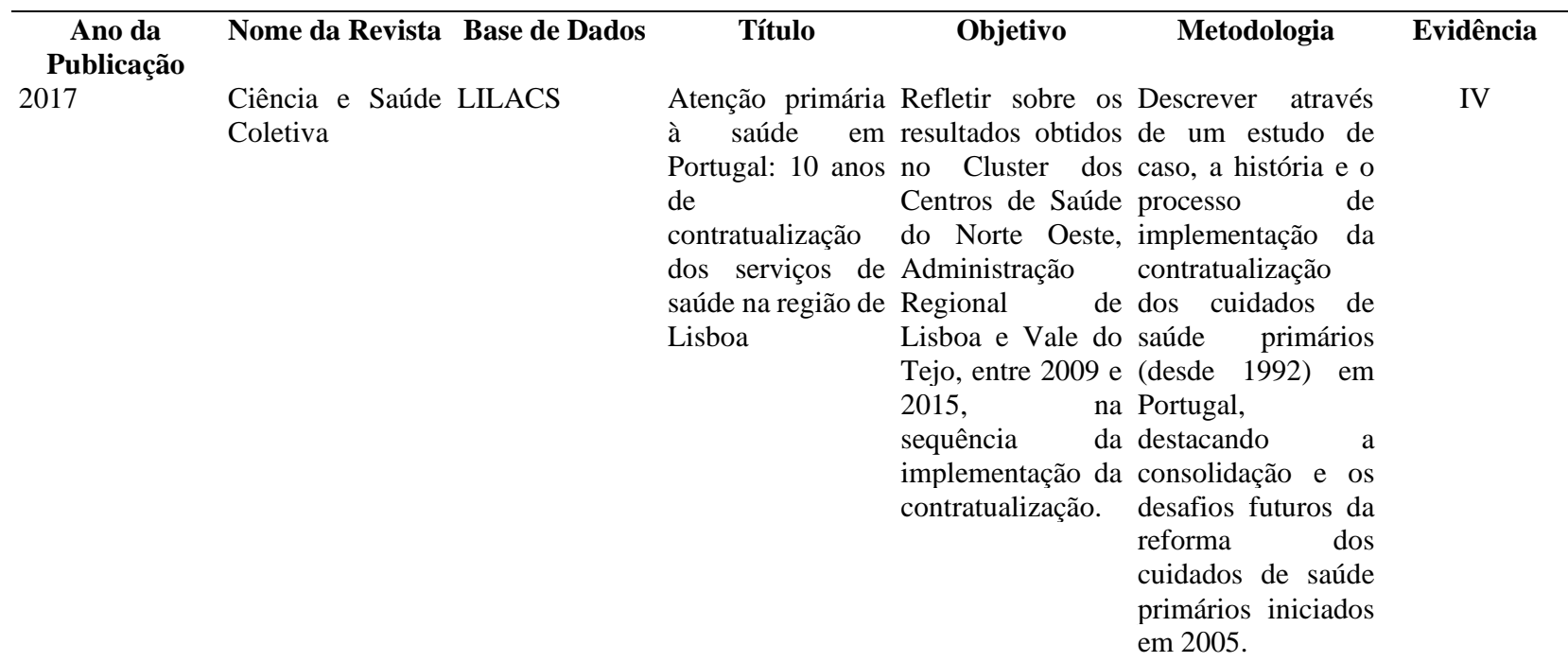


Research, Society and Development, v. 11, n. 3, e15811326265, 2022

(CC BY 4.0) | ISSN 2525-3409 | DOI: http://dx.doi.org/10.33448/rsd-v11i3.26265

2019

2019

2020

\author{
Caderno. Ibero- $\quad$ LILACS \\ americano. Dir. \\ Sanitário
}

Ciência \& Saúde LILACS Coletiva

Online Braz. J. BDENF Nursing

Revista Brasileira BDENF de Enfermagem

\author{
Auditoria em Analisar os Estudo quantitativo \\ descritivo, baseado \\ saúde e economia resultados da \\ em dados \\ da saúde: análise \\ de um estudo de \\ caso. \\ $\begin{array}{ll}\text { auditoria em } & \text { em dados } \\ \text { saúde na área de } & \text { secundários da }\end{array}$ \\ órteses, próteses e SES/RS, na área de \\ materiais \\ OPM e CMS. \\ especiais (OPM) e \\ cirurgias múltiplas \\ e sequenciais \\ (CMS), realizada \\ pela Secretaria \\ Estadual de Saúde \\ do Rio Grande do \\ Sul (SES/RS). \\ Avaliação de $\mathrm{O}$ objetivo deste Um estudo \\ tecnologias em estudo foi analisar descritivo foi \\ saúde no Brasil - os processos de realizado a partir de \\ uma perspectiva ATS no Brasil, uma revisão da \\ internacional. supervisionados literatura e análise \\ pela Comissão documental do \\ Nacional para a processo de ATS no \\ Incorporação de Brasil, Austrália, \\ Tecnologia em Canadá e Reino \\ Saúde Unido. \\ (CONITEC), e \\ comparar esses \\ processos com os \\ de países \\ considerados na \\ vanguarda neste \\ campo: Austrália, \\ Canadá e o Reino \\ Unido.
}

\section{Avaliação dos Analisar registros de qualidade enfermagem em registros pediatria: estudo enfermeiros descritivo}

Indicadores
efetividade
assistência
enfermagem
dimensão
segurança
paciente

de Validar
da
de eficácia
na
assistência
enfermagem
do hospitalar

dimensão
segurança
paciente.

a Estudo descritivo, dos transversal, de de análise documental. Realizou-se em auditoria entre julho de a outubro de 2019 extraindo dados referentes à assistência por meio da qualidade dos registros de enfermeiros

os Estudo quantitativo, de tipo survey, da mediante a técnica de Delphi eletrônica, com 52 na participantes selecionados pela do técnica Bola de Neve.
IV

IV

IV 
2020

Saúde

$\begin{array}{lrl}\text { Utilização } & \text { de } & \text { Conhecer } \\ \text { indicadores } & \text { de } & \text { dificuldades } \\ \text { qualidade: } & \text { estratégias } \\ \text { dificuldades } & \text { e } & \text { enfermeiros- } \\ \text { estratégias na voz líderes } & \text { lian } \\ \text { de enfermeiros- } & \text { utilização } \\ \text { líderes } & \text { indicadores } \\ & \text { qualidade } \\ & \text { ambiente } \\ & \text { hospitalar. }\end{array}$

A atuação da Descrever auditoria do atuação

Sistema Único em auditoria um estado Sistema Único de estado do Rio brasileiro Saúde em um Grande do Norte, estado brasileiro dividido em quatro
VI

III

VI

IV

IV

Fonte: Autores.

Conforme pode-se observar, a maioria dos estudos empreendeu em estudos descritivos. As evidências científicas mostraram-se no nível IV e no nível VI, respectivamente, o que reafirma que os objetivos dos artigos estão relacionados à validação, identificação e desenvolvimentos de processos que demonstrem ser eficazes, eficientes e efetivos no trabalho do 
auditor.

\section{Discussão}

Os resultados da pesquisa foram organizados em cinco categorias: anotações de enfermagem, educação em saúde, gestão da qualidade, protocolos para tecnologias em saúde e protocolos clínicos e indicadores de qualidade. Estes temas não se excluem e podem, inclusive, em muitos momentos mostrarem intersecções.

Através da análise dos artigos selecionados, foi possível extrair requisitos essenciais para o processo de auditoria, todavia observa-se, ainda, lacunas sobre o assunto investigado, ou seja, ainda pontos em que a discussão é necessária para maior desenvolvimento do segmento.

\section{Anotações de Enfermagem}

A auditoria hospitalar permite uma avaliação dos aspectos qualitativos e quantitativos relacionados à assistência. Também envolve a observação de aspectos organizacionais, operacionais e financeiros, com foco na qualidade dos cuidados prestados (Scarparo et al., 2008).

Porém, quando se discute a importância das anotações, deve-se considerar elementos limitantes, como a escassez de recursos humanos na enfermagem, bem como o ritmo de trabalho da enfermagem. De forma que, esses fatores dificultam o registro das informações, sendo, portanto, pontos diretos relacionados à melhoria da qualidade das anotações de enfermagem (Vituri et al., 2008).

Registrar de forma clara, completa e consistente é uma ação obrigatória e não voluntária, pois formaliza as ações do processo de trabalho da enfermagem, com a finalidade de respaldar o profissional e o paciente em caso de intercorrências envolvendo a assistência em saúde (Silva et al., 2020).

O registro em prontuário abrange diversos aspectos e respalda de forma ética e legal o profissional responsável pelo cuidado, assim como, o paciente. Em presença de registros incompletos, letra ilegível e inconsistente, além de não fazer diferencial algum para a equipe multidisciplinar durante o tratamento do paciente, pode influenciar em desperdícios e onerar os custos com a terapêutica para a instituição de saúde (Silva et al., 2021).

Cumpre salientar, que tal serve de apoio e a alta administração pois proporciona melhorias na gestão da qualidade, além de promoverem as mudanças imprescindíveis para o alcance de resultados (Siman et al., 2015).

\section{Educação em Saúde}

Um dos elementos imprescindíveis no processo de auditoria é o prontuário, importante ferramenta legal na avaliação da qualidade da assistência prestada ao cliente, fornecendo informações vitais para possíveis processos judiciais e convênios de saúde (Bazzanella et a., 2013).

É indispensável a presença de elementos como o conhecimento da equipe, a padronização do processo de trabalho com protocolos, a capacitação contínua e sistematizada, a reflexão sobre o conteúdo das informações e dos impressos para anotação. Sendo assim, o serviço da auditoria pode ser visto como um processo educativo, no qual não se busca responsáveis pela falha, mas, questiona o motivo e circunstâncias da não conformidade. Essa mudança de percepção estimula a participação da equipe na identificação e na resolução dos eventos adversos (Bandeira et al., 2015).

\section{Gestão da qualidade}

O profissional auditor contribui para a aplicação correta dos recursos públicos; avalia e acompanha a qualidade do serviço ofertado e a (in)satisfação do usuário; identifica irregularidades e fraudes, assim como recomenda ao gestor e órgãos competentes 
sugestões para as devidas providências; constata a necessidade de ampliação ou redução de serviços, conforme demandas de usuários; além de subsidiar os gestores, Conselhos de Saúde, Ministério Público, Judiciário, entre outros; avalia a gestão e emite pareceres, avalia contratos e atende demandas externas quando solicitado (Ministério da Saúde., 2013).

Outrossim, destaca-se que gestão de serviços de saúde é de fundamental importância, pois constitui uma prática administrativa que tem a finalidade de otimizar o funcionamento das organizações de forma a obter o máximo de eficiência (relação entre produtos e recursos empregados), eficácia (atingir os objetivos estabelecidos) e efetividade (resolver os problemas identificados) (Jorge et al., 2020).

Destaca-se que as organizações tem apropriado sistemas e habilidades para monitorar, rastrear e avaliar os resultados de uma auditoria (ambos antecipado e imprevisto), então as melhorias são mais prováveis de serem incorporadas e sustentadas (HutMossel et al., 2021).

Neste sentido, aventa-se que a governança clínica influenciou outros sistemas de saúde a definirem e implementarem políticas e diretrizes para assegurar a melhoria da qualidade da clínica, enfrentando, dentre outros fatores, a variabilidade na prestação de cuidados. A National Health Service - NHS propõem sete pilares para a governança clínica, sendo a efetividade clínica, auditoria clínica, gerenciamento de risco, uso da informação, educação e treinamento, gerenciamento de pessoas e envolvimento do paciente/público (Padilha, 2018).

Além disso, sinaliza-se que a governança clínica é um conjunto de práticas a serem aplicadas a todo o sistema de saúde, sendo a auditoria considerada como um ponto de relevante posição, pois, atua diretamente na melhoria dos profissionais, como também, no controle e padronização dos trabalhos, uma vez que, reforça os processos disciplinares e normativos na busca contínua para agregar valor à assistência, por meio de metodologias, protocolos e ferramentas de medição, a fim de garantir uma assistência de qualidade, além da efetividade nos custos, ou seja, empregando os recursos necessários evitando-se desperdícios (Oliveira, 2003).

\section{Protocolos para Tecnologias em Saúde e Protocolos Clínicos}

Protocolos são considerados importantes instrumentos para o enfrentamento de diversos problemas na assistência, na gestão dos serviços e dos custos.

Destarte, servem para orientar a utilização de diagnósticos e tratamentos que podem ser usados pelo médico no seu dia a dia, sendo ferramentas importantes para atualização na área da saúde e utilizados para reduzir a variação inapropriada na prática clínica. Cada protocolo clínico deve ser delineado para ser utilizado tanto no nível ambulatorial como hospitalar (Christensen et al., 2009).

Salienta-se que as diretrizes, para uma tecnologia, são recomendações preparadas de forma sistemática, com base em evidências científicas, com o propósito de influenciar as decisões dos profissionais de saúde e dos pacientes a respeito da atenção apropriada, em circunstâncias clínicas específicas (Báo et al., 2019).

Os protocolos para utilização de novas tecnologias, de alto custo e clínicos devem representar uma oportunidade, proporcionando qualidade ao acesso à saúde.

\section{Indicadores de Qualidade}

Os indicadores de qualidade são reconhecidos como ferramenta indispensável para o gerenciamento de boas práticas no ambiente hospitalar.

O controle dos indicadores por meio da gestão interna possibilita estratificar o tempo de internação, gerenciamento de leitos e o monitoramento assistencial sistemático, contribuindo para a gestão dos serviços de saúde e refletindo positivamente na qualidade da assistência bem como na diminuição dos custos hospitalares (Damasceno et al., 2020). 
A qualidade das informações, para a utilização dos indicadores, é de suma importância, visto que reflete o resultado do trabalho assistencial, além de permitir que as organizações monitorem seu desempenho e comparam-se com outras instituições, como foi visto em um estudo que desenvolveu indicadores de qualidade para pacientes com patologias agudas (Padilha, 2018).

\section{Conclusão}

Fica evidenciada a importância de ferramentas para a auditoria no processo de gestão das organizações em um ambiente globalizado e cada vez mais competitivo, pois são instrumentos que o auditor possui para atingir suas metas, agilizar a análise de resultados e gerar relatórios. Sendo assim, é essencial que as ações para a melhoria e criação de ferramentas sejam definidas, após terem sido planejadas e ter-se realizado um diagnóstico, por meio do levantamento das necessidades e mapeamento dos processos.

Visualiza-se que os registos de enfermagem são elementos fundamentais de gestão, sendo assim, devem garantir entre outros aspectos, o faturamento adequado da conta médico-hospitalar do paciente permitindo a manutenção das despesas inerentes à assistência oferecida pela instituição. Alguns desafios, ainda presentes neste contexto, exigem por parte da gestão, novos olhares e estratégias de conscientização.

Embora este estudo possua limitações temporais e geográficas das obras, acredita-se que as informações ora finalizadas possam servir como fulcro para futuras pesquisas, vislumbrando instigar enfermeiros auditores, instituições formadoras e de saúde a procurar cada vez mais estratégias para a efetiva implementação de ferramentas de gestão que possam garantir as competências necessárias à atuação nesta especialidade.

\section{Referências}

Bandeira, R. P; Nóbrega, M. M. D. A; Bezerra, A. M. F; Bezerra, W. K. T \& Pereira, D. S.(2015).O Papel do Enfermeiro na Auditoria Hospitalar. Revista Brasileira De Educação E Saúde, 5(4):11-16.

Báo, A. C. P; Amestoy, S. C; Moura, G. M. S. S \&Trindade, L. L.(2019). Quality indicators: tools for the management of best practices in Health. Rev Bras Enferm.,72(2):360-6.

Bardin, L.(2011). Análise de conteúdo. São Paulo: Edições 70.

Bazzanella, A. L. N \& Slob, E. (2013). A Auditoria como Ferramenta de Análise para a Melhoria da Qualidade no Serviço Prestado. Caderno Saúde e Desenvolvimento,.3(2):1-16.

Botelho, L. L. R; Cunha, C. C. A \& Macedo, M. O. (2011). Método Da Revisão Integrativa Nos Estudos Organizacionais, 5(11):121-36.

Brasil. Ministério da Saúde.(2013). Portaria 3.390, de 30 de dezembro de 2013. Política Nacional de Atenção Hospitalar (PNHOSP) no âmbito do Sistema Único de Saúde (SUS), estabelecendo-se as diretrizes para a organização do componente hospitalar da Rede de Atenção à Saúde (RAS). Brasília, DF.

Carvalho, A. I \& Barbosa, P.R. (2010). Políticas de Saúde: fundamentos e diretrizes do SUS.Florianópolis: UFSC; Brasília: CAPES.

Christensen, C. M; Grossman, J. H. \& Hwang, J. (2009). Inovação na Gestão de saúde.: a receita para reduzir custos e aumentar a qualidade. Porto Alegre: Bookman.

Damasceno, V. A; Alves, K. K. A. F. (2020). Aplicação e contribuições dos indicadores hospitalares: uma revisão integrativa. Research, Society and Development, 9(8):e902986637.

Galvão, C. M. (2006). Níveis de evidência. Acta Paul Enferm., 19(2), 5-5.

Hut-Mossel, L.; Ahaus, K.; Welker, G. \& Gans, R.(2021).Understanding how and why audits work in improving the quality of hospital care: A systematic realist review. Plos One, 16(3): e0248677.

Jorge, M. S. B.; Carvalho, M. R. R. \& Costa, L. S. P.(2020).Gestão em saúde dos serviços médicos de emergência: uma revisão escopo. Research, Society and Development, 9(11): e95591110703.

Luongo, J. (2011). Gestão da qualidade em saúde. 1ed -São Paulo: Rideel.

Moher, D.; Liberati, A.; Tetzlaff, J \& Altman, D.G.(2009).The PRISMA Group 2009. Preferred reporting items for systematic reviews and meta-analyses: the PRISMA Statement. PLoS Med, 6(6):e1000097. 
Research, Society and Development, v. 11, n. 3, e15811326265, 2022

(CC BY 4.0) | ISSN 2525-3409 | DOI: http://dx.doi.org/10.33448/rsd-v11i3.26265

Oliveira, A. F.(2003).Avaliação do impacto das intervenções nos pontos críticos detectados na pré-análise de auditoria em enfermagem. Tese. São Paulo.

Oxford Brazil. (2018). Critical Appraisal Skills Programme (CASP).

Padilha, R. Q. (2018). Princípios para a gestão da clínica: conectando gestão, atenção à saúde e educação na saúde. Ciênc. saúde coletiva, 23(12):4249-4257.

Peron, C. (2021). Auditoria e Pacto de Gestão: Perspectivas e Desafios. Artigonal - Diretório de Artigos Gratuitos.

Scarparo, A. F \& Ferraz, C. A. (2008). Auditoria em Enfermagem: identificando sua concepção e métodos. Rev. Bras. Enferm., 61(3): 302-305.

Scarparo, A. F; Clarice Aparecida Ferraz, C. A; Chaves, L. D. P \& Gabriel, C. S. (2010). Tendências da função do enfermeiro auditor no mercado em saúde. Texto \& Contexto - Enfermagem, 19(1):85-92.

Silva, L. C. S.; Prado, M. A.; Carneiro, L. C.; Filho, A. V. M.; Costa, T. A. M.; Ribeiro, D. P. O; Bezerra, A.L.Q \& Bezerra, M. A. (2021).Qualidade dos registros de enfermagem em um hospital: auditoria. Research, Society and Development, 10(10):e229101018684.

Silva, L. C. S.; Prado, M. A.; Fernandes, M. R.; Filho, A. V. M.; Sousa, M. C.; Costa, T. A. M. et al. (2021). Inconformidades nos registros em prontuários: opinião dos trabalhadores de saúde. Research, Society and Development, 10(12): e294101220587.

Siman, A. G; Cunha, S. G. S; Martins, E. S \& Brito, M. J. M.(2015).Estratégia do trabalho gerencial para alcance da acreditação hospitalar. Rev Min. Enferm.,19(4):815-22.

Sousa, M. C.; Costa, T. A. M. et al. (2021). Inconformidades nos registros em prontuários: opinião dos trabalhadores de saúde. Research, Society and Development, 10(12): e294101220587.

Tricco, A. C., Lillie, E., Zarin, W., O’Brien, K. K., Colquhoun, H., Levac, D., et al. PRISMA Extension for Scoping Reviews (PRISMA-ScR): checklist and explanation. Annals of Internal Medicine, 169(7), 467-473.

Vituri, D. \& Matsuda, L.(2008). Analysis of nursing records as to care quality: a retrospective descriptive exploratory study of quantitative approach. Online Braz. J Nurs.,14; 7(1). 